\title{
pH-Driven Reversible Self-Assembly of Micron-Scale DNA Scaffolds
}

\author{
Leopold N. Green, ${ }^{\dagger, \|}$ Alessia Amodio, ${ }^{\S, \|}$ Hari K. K. Subramanian, ${ }^{\ddagger}$ Francesco Ricci, ${ }^{*} \S(0)$ \\ and Elisa Franco $*+\neq 0$ \\ ${ }^{\dagger}$ Department of Bioengineering and ${ }^{\ddagger}$ Department of Mechanical Engineering, University of California, Riverside, California 92521, \\ United States \\ ${ }^{\S}$ Department of Chemical Science and Technologies, University of Rome, Tor Vergata, Via della Ricerca Scientifica 00133, Rome, \\ Italy
}

Supporting Information

ABSTRACT: Inspired by cytoskeletal scaffolds that sense and respond dynamically to environmental changes and chemical inputs with a unique capacity for reconfiguration, we propose a strategy that allows the dynamic and reversible control of the growth and breakage of micronscale synthetic DNA structures upon $\mathrm{pH}$ changes. We do so by rationally designing a $\mathrm{pH}$-responsive system composed of synthetic DNA strands that act as $\mathrm{pH}$ sensors, regulators, and structural elements. Sensor strands can dynamically respond to $\mathrm{pH}$ changes and route regulatory strands to direct the self-assembly of structural elements into tubular structures. This example represents the first demonstration of the reversible assembly and disassembly of micron-scale DNA scaffolds using an external chemical input other than DNA. The capacity to reversibly modulate nanostructure size may promote the development of smart devices for catalysis or drug-delivery applications.

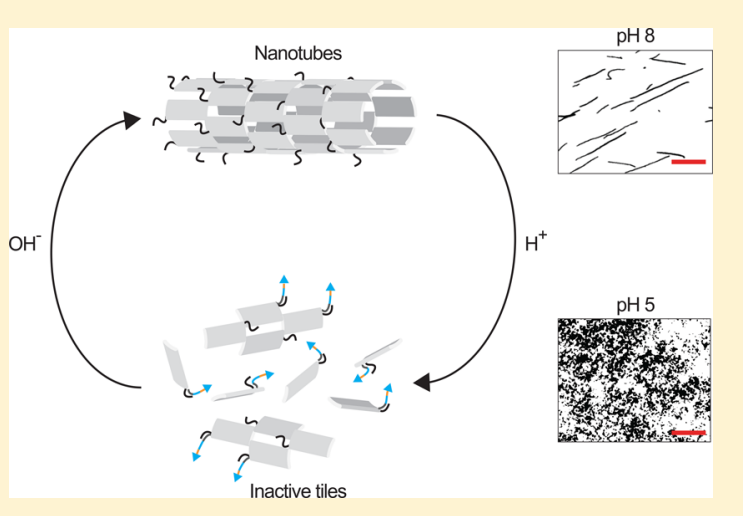

KEYWORDS: DNA nanotechnology, nucleic acids, dynamic self-assembly, $p H$, active scaffolds

B iological cells adapt their shape in response to environmental and chemical stimuli through the integration of sensing elements, signal processing circuits, and self-assembling components such as microtubules and actin filaments. ${ }^{1}$ Mimicking this architecture, artificial programmable materials with the responsiveness of cytoskeletal scaffolds may be obtained by rationally designing self-assembly processes regulated by upstream components that continuously sense the environment. Nucleic acids are one of the most promising substrates with which to pursue this endeavor due to the programmability of Watson-Crick base pairing, which has enabled the development of self-assembling scaffolds at the nano- and micron-scale, ${ }^{2}$ as well as sensors, ${ }^{3}$ and informationprocessing circuits. ${ }^{4}$

One of the first demonstrations of DNA self-assembly was achieved using DNA tiles built by stacking two to three DNA helices using multiple Holliday junctions or crossovers (Figure 1a, left). Tiles can be viewed as monomers that interact via single stranded domains, or sticky ends, to form lattices or tubular structures. ${ }^{5-7}$ Such DNA tiling systems are an ideal candidate for building micron-scale reconfigurable scaffolds, due to the simplicity of their programmable interactions. For example, by choosing correctly the length of the tile sticky ends, it has been possible to build a variety of DNA nanotubes with distinct patterning and chirality. ${ }^{7-9}$ The structural and mechanical features of these DNA nanotubes are close to those of microtubules and actin filaments, and they are amenable to functionalization with a variety of ligands. ${ }^{10-12}$ Individual DNA nanotubes can reach dozens of microns in length and rank among the largest DNA structures; thus, they could be used as exogenous scaffolds in biological and artificial cells, allowing for precise localization of thousands of ligands relevant for optics, catalysis and drug delivery purposes. ${ }^{13,14}$ However, size may limit the use of DNA nanotubes in biomedical applications because it could decrease their diffusion and uptake by cells and organelles. ${ }^{15}$ This limitation could be overcome by developing nanotube variants whose assembly and disassembly pathways can be controlled reversibly and isothermally, making it possible to switch between rapidly diffusing nanoscale tiles and robust micron-scale structures on demand.

Several approaches have been proposed to control the assembly and disassembly of DNA nanostructures. For example, it has been recently demonstrated that by using DNA strands modified with light-sensitive groups, it is possible to assemble DNA nanostructures of different complexity upon light irradiation. ${ }^{16,17}$ Similarly, other approaches have been proposed in which different environmental, biological, or chemical inputs can be used to control the assembly of DNA-based nanoscale structures. ${ }^{18,19}$ Because the role of $\mathrm{pH}$ is

Received: July 1, 2017

Revised: November 20, 2017 


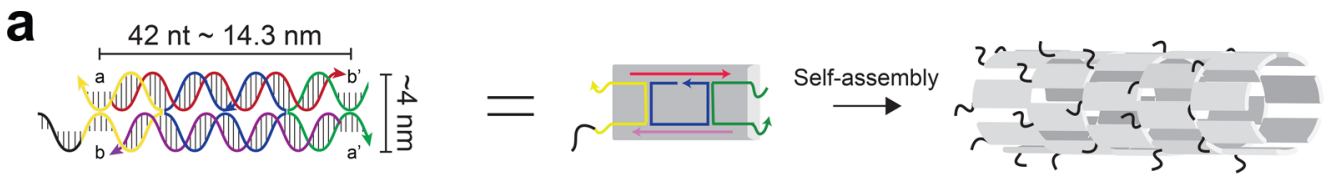

b

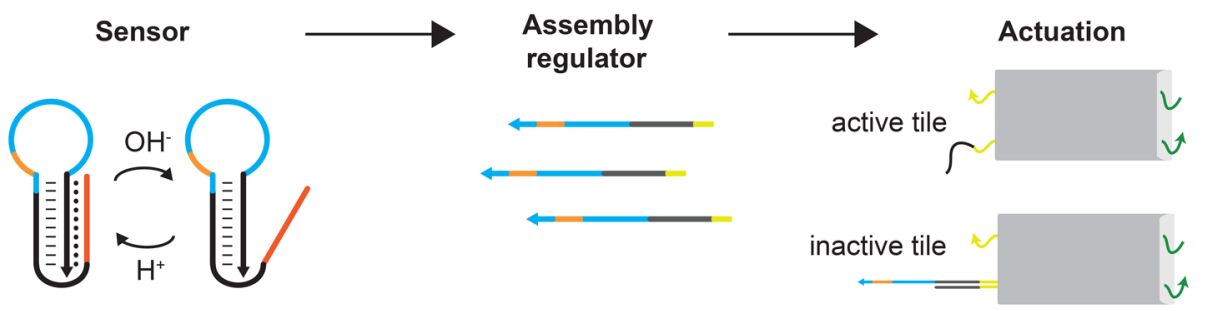

C

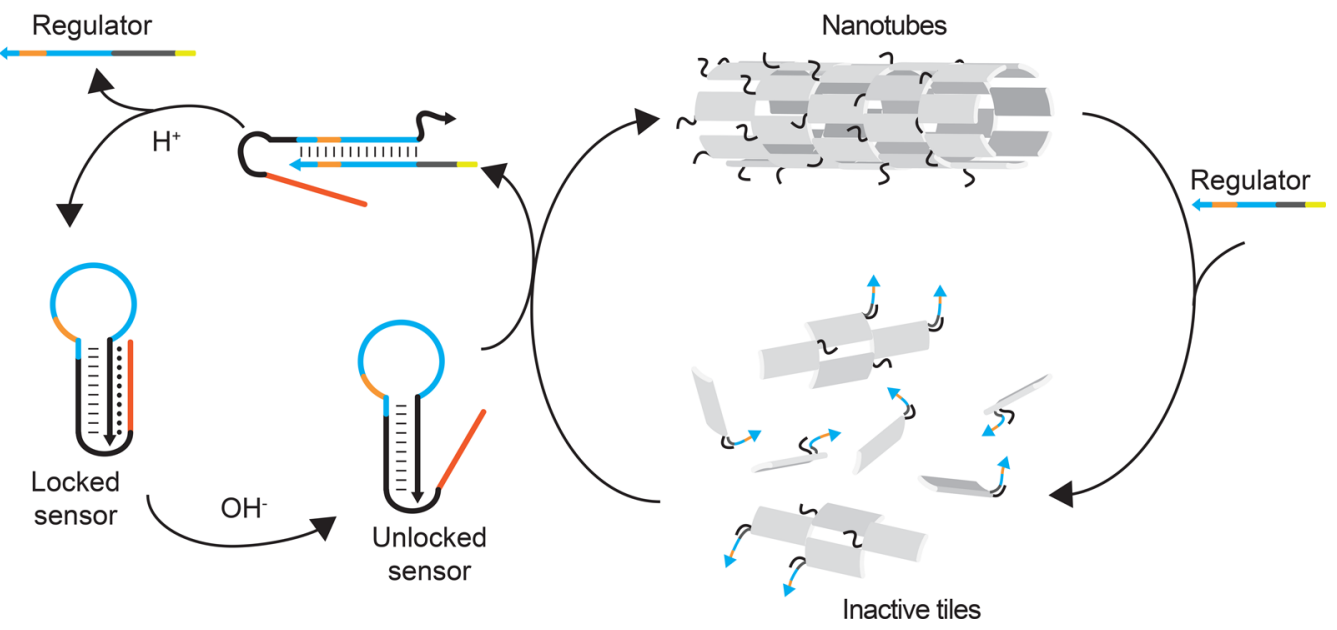

Figure 1. Modular approach to obtain pH-directed reversible isothermal control of DNA nanotube assembly and disassembly. (a) Left: double crossover, antiparallel helices, even number of half-turns between intratile crossovers, and even number of helical half-turns between intertile crossovers (DAE-E) tiles assemble from five DNA strands (each strand has a distinct color) forming two double helices held together by Holliday junctions (crossovers); our tiles were re-engineered to include a toehold domain. ${ }^{7,34}$ Tiles bind via sticky-end domains (a, $a^{\prime}, b$, and $\left.b^{\prime}\right)$ and assemble into nanotubes; nanotubes form instead of lattices due to the chosen intertile crossover distance. Middle: abstraction of tile with toehold. Right: abstraction of assembled tiles forming nanotubes where toeholds are exposed by design on the external surface. (b) To control the assembly and disassembly of DNA tiles, we used $\mathrm{pH}$-sensing elements that release or sequester regulator strands, which, in turn, activate or deactivate the tiles by interacting with the toeholded sticky end. (c) Reaction mechanisms: the pH-responsive sensor can be in a locked (low $\mathrm{pH}$ ) or unlocked (high $\mathrm{pH}$ ) state depending on the closed or open conformation of the triplex-forming domain. When the sensor is unlocked, it displaces and sequesters the regulator strand, thereby promoting nanotube assembly. When the sensor is locked, the regulator is released in solution and binds to the tiles, deactivating them.

of paramount importance for living organisms (i.e., protons dictate the charge and structure of macromolecules and are an integral part of many metabolic pathways), ${ }^{20,21}$ being able to reversibly control the functionality of different DNA nanostructures using $\mathrm{pH}$ as the triggering input could have intriguing potential applications in drug-delivery research. ${ }^{22}$ We and other groups have for example recently demonstrated that the employment of $\mathrm{pH}$-sensitive DNA triplex motifs based on Hoogsteen interactions allows us to control DNA-based sensing platforms and molecular switches, ${ }^{23-25}$ to develop $\mathrm{pH}$ responsive systems and materials, ${ }^{26-28}$ to drive the load and release of molecular cargoes, ${ }^{29}$ to design stimuli-responsive DNA-based hydrogels, ${ }^{30}$ to trigger the aggregation and disaggregation of nanoparticles, ${ }^{31,32}$ and to initiate the assembly of DNA tiles. ${ }^{33}$ However, the possibility to use $\mathrm{pH}$ as an input to control isothermally and reversibly the assembly of micronsized DNA nanostructures has never been explored.

Motivated by the above arguments and building on the availability of programmable DNA nanotubes and tunable $\mathrm{pH}$ sensitive DNA domains, we designed a scheme for reversible breakage and growth of DNA nanotubes upon $\mathrm{pH}$ changes that relies on the interaction of three main elements: (1) DNA tiles, (2) DNA strands that regulate assembly, and (3) DNA strands that sense $\mathrm{pH}$. As a building block to assemble nanotubes, we employed the DAE-E tile type, ${ }^{7,9}$ which consists of five distinct strands of DNA (Figure 1a, left); the acronym indicates Double crossover, Antiparallel helices, Even number of half-turns between intra-tile crossovers, and Even number of helical half-turns between inter-tile crossovers. The formation of individual tiles requires thermal annealing; however, nanotube assembly proceeds at room temperature ${ }^{7,8,34}$ (Figure 1a, right). We redesigned such DNA tiles to include an actuation domain that allows us to activate or deactivate their capacity to selfassemble $^{35}$ (Figure 1a, b). These actuation domains are singlestranded toeholds exposed by design on the external nanotube surface (black domains; Figures la and S1); these nanotubes have a diameter of 6-10 tiles, which indicates that their diameter is not significantly affected by the actuation domains ${ }^{7}$ 


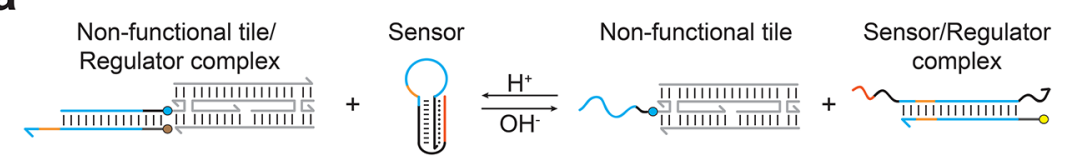

b
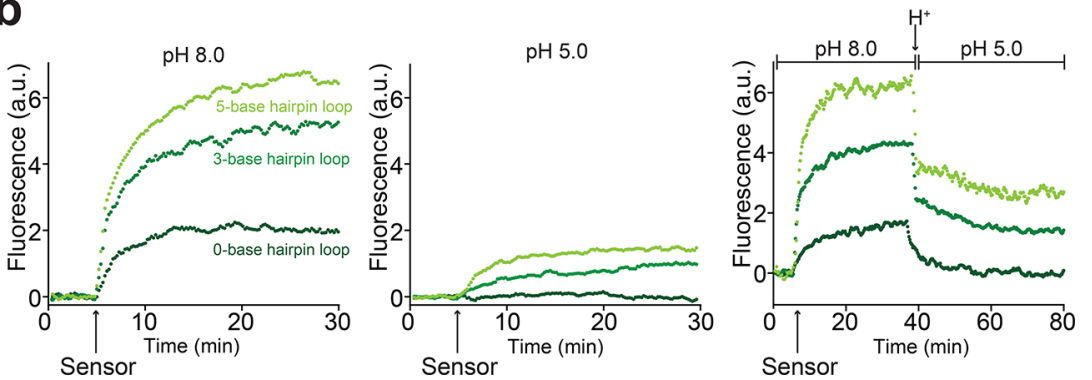

Figure 2. Characterization of $\mathrm{pH}$-induced tile activation and deactivation as a function of the length of the hairpin loop toehold domain. (a) Expected interactions of $\mathrm{pH}$-responsive sensor, regulator, and nonfunctional tiles. Binding of the sensor and regulator strands initiates via a toehold domain, part of which is included in the sensor hairpin loop (orange domain). When the sensor is locked, the regulator binds to the nonfunctional tile making it inactive (low fluorescence). When the sensor is unlocked, it is expected to displace the regulator from the tile (high fluorescence). (b) Fluorimetry results showing the behavior of three sensor variants presenting a toehold domain zero, three, or five bases long included in the sensor hairpin loop (0TH-10S, 3TH-10S, and 5TH-10S, respectively). Left: at $\mathrm{pH} 8.0$ (basic), the sensor is unlocked and displaces and sequesters the regulator; tiles are in their active state. Center: at pH 5.0 (acidic), the sensor is locked, and thus, regulator is available to bind to tiles and switch them to their inactive state. Right: Switching from $\mathrm{pH} 8.0$ to $\mathrm{pH}$ 5.0, the regulator is released from the regulator and sensor complex and can bind to the tiles, inactivating them.

(Figure S35). Single-stranded DNA sequences acting as assembly regulators are designed to interact with the toehold (actuation domain) on the tiles and invade the sticky-end bond between two tiles, causing the rapid disassembly of DNA nanotubes and the inactivation of individual tiles (Figures $1 \mathrm{~b}$ and S3 and Supporting Information section 2.4). Finally, we rationally designed a DNA switch or sensor that can dynamically respond to $\mathrm{pH}$ changes. This sensor consists of two major domains. One domain is complementary to the regulator strand and is used to displace the regulator from the tiles, allowing them to become active and assemble into nanotubes (Supporting Information section 2.4 and Figure S4). The other domain is a $\mathrm{pH}$-sensitive, triplex-forming stem, which, under acidic pHs, is able to form an intramolecular triplex hairpin that inhibits the ability of the sensor to hybridize with the regulator. Under these conditions, regulators can freely inactivate the tiles and disassemble the nanotubes (Supporting Information section 2.5). At basic $\mathrm{pH}$, Hoogsteen interactions in the triplex structure are destabilized, and the sensor is "unlocked"; this facilitates regulator and sensor interactions, and enables the sequestration and displacement of the regulator from tiles, which become active and self-assemble into nanotubes (Figure 1c).

With the goal of tuning the efficiency of regulator displacement we first designed three variants of the $\mathrm{pH}$ sensor in which the length of the triplex-forming stem was fixed (i.e., 10 bases), while the exposed portion of the domain complementary to the regulator toehold, ${ }^{36}$ placed within the sensor hairpin loop (Figures $1 \mathrm{~b}$ and $2 \mathrm{a}$, orange domains), was varied to be zero, three, or five bases long for variants we named $0 \mathrm{TH}-10 \mathrm{~S}$, 3TH-10S, and 5TH-10S, respectively. We initially characterized the response of the $\mathrm{pH}$ sensor using bulk fluorescence spectroscopy (Figure 2a,b). To monitor the interactions between the actuator domains (on the tiles), regulators, and sensors, we labeled the regulator with a fluorophore and a nonfunctional variant of tiles that include actuator domains with a quencher (these tiles cannot assemble into nanotubes because they contain only one of the four sticky ends). First, we tested regulator displacement upon the addition of the sensor in an equimolar solution of tile and regulator at basic $\mathrm{pH}$ (i.e., $\mathrm{pH}=8.0$ ). At this $\mathrm{pH}$, the destabilization of Hoogsteen interactions brings the sensor to its unlocked form, and efficient displacement of the regulator from the tile is observed (Figure $2 b$, left). As expected, the efficiency of displacement increases as we increase the length of the hairpin-loop domain complementary to the toehold of the regulator. More specifically, we observe that regulator displacement is most efficient when using sensor variants $3 \mathrm{TH}-10 \mathrm{~S}$ and 5TH-10S (hairpin loop containing three and five bases of the regulator toehold complement); only partial tile activation is obtained using variant $0 \mathrm{TH}-10 \mathrm{~S}$ (no regulator toehold complement bases in the hairpin loop). At $\mathrm{pH}$ 5.0, in contrast, triplex formation locks the sensor and inhibits displacement of the regulator from the tile (Figure $2 b$, center). Of note, while sensor 0TH-10S shows a complete inhibition of the displacement reaction, tile activation leak is observed with sensors $3 \mathrm{TH}-10 \mathrm{~S}$ and 5TH-10S. The data shown in Figure $2 \mathrm{~b}$ were further processed and normalized to estimate the fraction of regulator that is displaced from tiles; our normalization suggests that sensor variants $3 \mathrm{TH}-10 \mathrm{~S}$ and $5 \mathrm{TH}-10 \mathrm{~S}$ can displace about $30-40 \%$ of the regulator from tiles (Figure S42 and Supporting Information section 4.7). A control experiment carried out using a $\mathrm{pH}$ sensor having the same sequence of $3 \mathrm{TH}-10 \mathrm{~S}$ but lacking the triplex-forming domain (the 5 ' overhang domain has been substituted with a random sequence) shows that, as expected, the displacement process is independent of $\mathrm{pH}$ in the absence of the $\mathrm{pH}$-dependent triplex domain (Figure S18).

We then tested whether the sensor-regulator strand displacement reaction can be reversibly controlled by $\mathrm{pH}$ changes. To do so, we initiated our experiments maintaining the sensor elements in unlocked state at $\mathrm{pH} 8.0$ (Figure $2 \mathrm{~b}$, right), thus producing optimal regulator displacement. Upon decreasing the solution $\mathrm{pH}$ (to $\mathrm{pH} \mathrm{5.0)}$ we were able to reverse the stranddisplacement process and observe an immediate decrease of fluorescence associated with the formation of the tile and regulator complex (Figure $2 b$, right). Displacement is reversible 
a

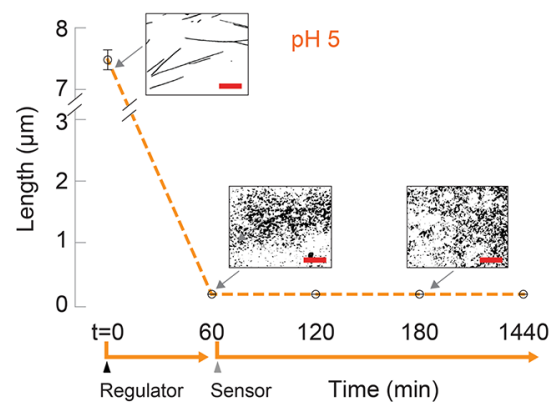

b

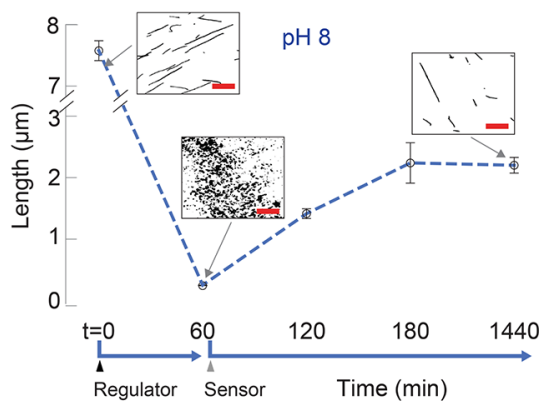

C

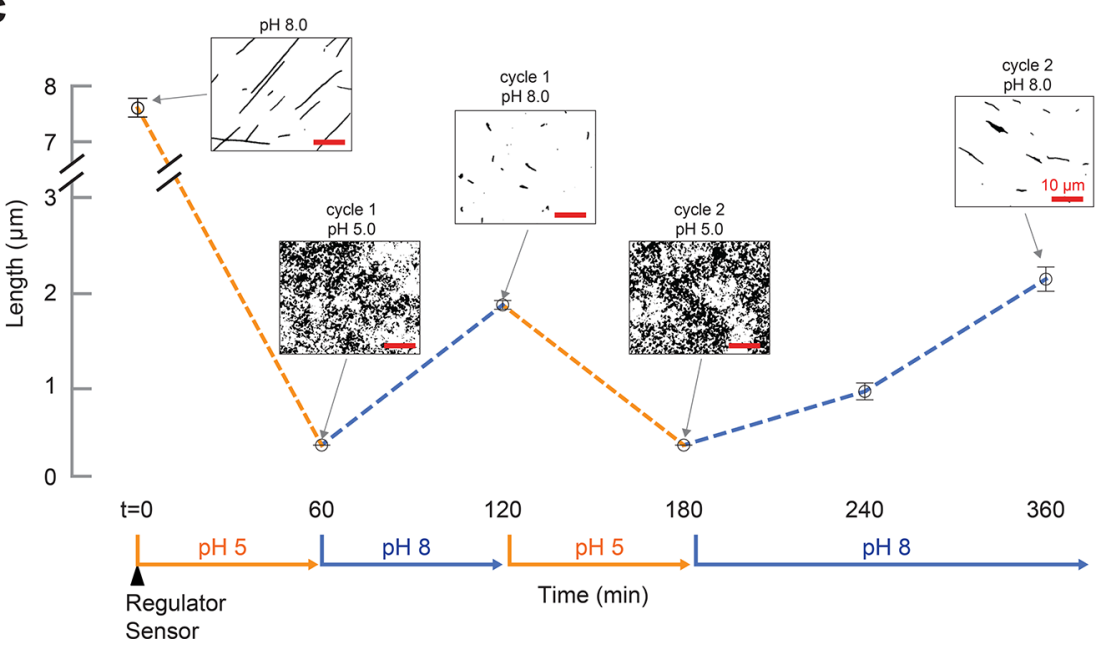

Figure 3. pH-responsive sensors and regulators achieve reversible isothermal assembly and disassembly of DNA nanotubes. We tested the performance of sensor variant $3 \mathrm{TH}-10 \mathrm{~S}$ in routing self-assembly of DNA nanotubes. Each plot shows the mean and standard deviation of the mean (computed over triplicate experiments) of nanotube length distributions, as well as example binary fluorescence microscopy images of the sample at different times (example raw images are shown in Figure S30). (a) When incubated at pH 5.0, nanotubes disassemble in the presence of regulator but do not reassemble upon the addition of a sensor, which is in a locked state. (b) When incubated at $\mathrm{pH}$ 8.0, nanotubes disassemble in the presence of regulator and reassemble in the presence of sensor, which is in an unlocked state. (c) We monitored the mean nanotube length, while the solution $\mathrm{pH}$ was switched from high to low twice (two subsequent cycles). Both 3TH-10S sensor and regulator strands are present in solution at all times during the experiment. When the sample is in acidic conditions, the sensor is locked, and the regulator is released, resulting in complete nanotube breakage; when the sample is in basic conditions, the sensor is unlocked, the regulator is displaced and sequestered from tiles, and nanotube growth is promoted.

in all three variants; however, variant $5 \mathrm{TH}-10 \mathrm{~S}$ exhibits a significant baseline fluorescence after addition of $\mathrm{H}^{+}$, suggesting that a fraction of regulator is still sequestered by the sensor. In contrast, data indicate that when using sensor $0 \mathrm{TH}-10 \mathrm{~S}$, a larger fraction of regulator strands is released after addition of $\mathrm{H}^{+}$; however, this variant also shows minimal regulator sequestration at high $\mathrm{pH}$. These results suggest that variant $3 \mathrm{TH}-10 \mathrm{~S}$ is the best to achieve reversible sequestration and release of the regulator.

We thus used such a $\mathrm{pH}$-dependent sensor (variant $3 \mathrm{TH}$ $10 \mathrm{~S})$ to reversibly control the assembly and disassembly of DNA nanotubes. Using fluorescence microscopy (one of the tile strands is labeled with $\mathrm{Cy} 3$ ), we characterized changes in the mean length of DNA nanotubes caused by changes in $\mathrm{pH}$, which was varied over time between acidic $(\sim \mathrm{pH} 5.0)$ and basic $(\sim \mathrm{pH} 8.0)$. Fluorescence microscopy images were processed using an in-house MATLAB script to obtain length distributions, which are reported in section 4 of the Supporting Information as violin plots; from the measured length distributions, we computed the mean length and the standard deviation of the mean over triplicate experiments. This approach allows us to rapidly collect quantitative length statistics of nanotubes, although structures below $0.33 \mu \mathrm{m}$ are neglected due to limited microscope resolution. Small assemblies could be qualitatively assessed using atomic force microscopy (AFM); see, for instance, Supporting Information section 4.5.4.

First, we verified that in the absence of the regulators, nanotubes do not spontaneously disassemble when incubated at $\mathrm{pH} 5.0$ or $\mathrm{pH} 8.0$ (Supporting Information section 4.1). In both conditions, the mean length of DNA nanotubes remains stable (with an average length between 5 and $7 \mu \mathrm{m}$ ) after annealing and incubation, consistently with earlier observations at the standard $\mathrm{pH}$ of TAE $12 \mathrm{mM} \mathrm{Mg}$ buffer. ${ }^{8,37}$ When the regulator is added at a $1 \mu \mathrm{M}$ concentration ( $1 \times$ relative to tiles concentration in solution), nanotubes disassemble within $1 \mathrm{~h}$ and remain broken for over $24 \mathrm{~h}$ of incubation at both $\mathrm{pH} 5.0$ and $\mathrm{pH} 8.0$ (Supporting Information section 4.2 and Figures S11 and S12).

The addition of sensor strand to a solution containing both tiles and regulators (nanotubes are broken) at $\mathrm{pH} 5.0$ does not yield nanotube growth for over $24 \mathrm{~h}$, as expected (Figure 3a). A minimal level of leakage reaction is observed in the $5 \mathrm{TH}-10 \mathrm{~S}$ variant, in which nanotubes moderately regrow reaching $1 \mu \mathrm{m}$ 
mean length after $24 \mathrm{~h}$ (Figure S21 and Supporting Information section 4.3). Conversely, the addition of the same sensor element at $\mathrm{pH} 8.0$ leads to a quick regrowth of the nanotubes that reach a mean length of $2-3 \mu \mathrm{m}$ within $2 \mathrm{~h}$ (Figure $3 \mathrm{~b}$ ). We note that this is about $60 \%$ lower than the post-anneal mean length. This could be due to low efficiency of regulator displacement from tiles even when the sensor is fully unlocked; this hypothesis is supported by the fact that when normalized, fluorescence spectroscopy data suggest that the most efficient sensors can successfully displace roughly 30$40 \%$ of the regulator strand from tiles, thereby leaving most tiles inactive (Figure S42 and Supporting Information section 4.7). It is also possible that tiles become kinetically trapped in a complex that includes tile, regulator and sensor elements, making tiles unavailable for polymerization. An overview of experiments conducted with all the sensor variants is in the Supporting Information section 4.3. Agarose gel electrophoresis (Figure S33 and Supporting Information section 4.5.3) and AFM images (Figure S34 and Supporting Information section 4.5.4) corroborate the results of fluorescence microscopy assays. AFM images of nanotubes incubated at $\mathrm{pH} 5.0$ in the presence of regulator show that the sample includes small loose lattices but no discernible nanotubes (Figure S34).

Lastly, we tested the possibility to achieve two consecutive cycles of nanotube assembly and disassembly upon consecutive $\mathrm{pH}$ changes. We first added both regulator and sensor strands to a nanotube solution at $\mathrm{pH}$ 5.0, and we observed completely broken nanotubes after $1 \mathrm{~h}$ of incubation (Figure 3c). We then increased the $\mathrm{pH}$ of the solution to 8.0 and measured a mean length of nanotubes of $1 \mu \mathrm{m}$ after $1 \mathrm{~h}$, consistent with previous control experiments (Figure $3 \mathrm{~b}$ ). We then changed the solution $\mathrm{pH}$ to acidic and then basic for one more complete cycle of nanotube breakage and reassembly and observe efficient and reversible breakage and growth of nanotubes (Figure 3c).

The pH-sensor element domains can be rationally engineered to direct nanotube assembly and disassembly upon $\mathrm{pH}$ changes. For example, we designed shorter sensor variants by reducing the length of the triplex stem to 8 and 7 bases, maintaining an exposed toehold domain of two and three bases, respectively (variants $2 \mathrm{TH}-8 \mathrm{~S}$ and $3 \mathrm{TH}-7 \mathrm{~S}$, Figure S2d,e and Supporting Information section 4.4). With $\mathrm{pH}$-sensor variant $2 \mathrm{TH}-8 \mathrm{~S}$, we achieved two nanotube disassembly and reassembly cycles (see Figure S32) that are comparable with those obtained with variant 3TH-10S, shown in Figure 3c. Notably, variant 3TH-7S exhibits a leaky behavior, comparable to that of variant 5TH-10S (nanotubes reach average length of $2.5 \mu \mathrm{m}$ after $24 \mathrm{~h}$ of incubation at $\mathrm{pH} 5.0$; Figure S28), suggesting that a short exposed toehold domain and a long stem are necessary to contain the leak.

We have illustrated an approach to reversibly control assembly and disassembly of micron-scale DNA nanostructures using $\mathrm{pH}$ as a canonical environmental cue. We have done so by controlling the activity of nanoscale monomers via $\mathrm{pH}$ dependent DNA motifs operating as sensors and regulators of the assembly process. The dynamic control of assembly was demonstrated to be reversible and to operate at constant temperature. We have characterized the $\mathrm{pH}$ response of the sensors and regulators as a function of relevant domains and characterized the corresponding efficiency of nanotube disassembly and reassembly. Our work highlights some important trade-offs for the design of efficient $\mathrm{pH}$-dependent self-assembling systems. A longer exposed toehold domain in the $\mathrm{pH}$ sensor increases the speed and efficiency of regulator displacement (promoting reassembly) but can also introduce a leak in conditions at which regulator displacement should not occur (acidic $\mathrm{pH}$ and locked sensor); this leak can be mitigated by increasing the length of the triplex stem. We also found that, in our particular system, nanotube regrowth after disassembly is slow, and the nanotube mean length does not reach its prebreakage value. This is likely due to two phenomena: a significant fraction of the sensor may not become fully unlocked upon switching from $\mathrm{pH} 5.0$ to $\mathrm{pH} 8.0$; thus, a large fraction of regulator cannot be displaced from the tiles that remain inactive. A control experiment shows that disassembled nanotubes can regrow achieving their original mean length upon addition of an excess amount of "fresh" sensor that was not previously locked (Supporting Information section 4.6.3). The second phenomenon hindering regrowth may be the formation of intermediate inactive complexes that trap sensor, regulator strand and tiles, thereby reducing the nanotube polymerization rate. This accumulation of inert complexes might also be an obstacle for disassembly, and indeed preliminary attempts to achieve more than two cycles of disassembly (switching the solution to $\mathrm{pH}$ 5.0) were not successful. These phenomena cannot be mitigated by varying the tile concentration or the $\mathrm{Mg}^{2+}$ concentration (Supporting Information section 4.6). By improving the design of sensor and regulator molecules, it will likely be possible to improve the efficiency of tile deactivation and activation, and thus increase the reversibility of assembly and disassembly.

Our results are a very promising step toward the development of active DNA nanomaterials capable of sensing and reconfiguration. The approach we described is modular because it relies on the interconnection of sensing, regulation, and actuation components to direct self-assembly. The sensing and regulation elements can be engineered in isolation with respect to the DNA nanostructure and made responsive to a chemical or physical signals other than $\mathrm{pH}$. Regulators can then be matched to actuation domains (toeholds) on the target DNA nanostructure. Virtually any aptamer could be used as a sensing domain paired to self-assembly regulators that may be activated or deactivated depending on the presence or absence of the aptamer target. Thus, it is possible to adapt our architecture to trigger assembly and disassembly of DNA scaffolds using an expandable range of chemical or physical cues. Because DNA nanostructures can serve as scaffolds for a variety of ligands, the proposed framework could be used to modularly control the organization of heterogeneous nanomaterials with a variety of functions. ${ }^{38,39}$ We also speculate that the capacity to reversibly modulate nanostructure size may promote the development of smart devices for catalysis or drug delivery. For instance, tiles could permeate, diffuse, and assemble into a variety of environments otherwise inaccessible to large scaffolds, such as inside cells or cellular organelles, where they could spatially organize components and modulate efficiency of metabolic pathways. Conversely, nanotubes could be robust vectors for molecular cargo, which disassemble near a target and reassemble on demand.

\section{ASSOCIATED CONTENT}

\section{S Supporting Information}

This material is available free of charge at The Supporting Information is available free of charge on the ACS Publications website at DOI: 10.1021/acs.nanolett.7b02787. 
Additional details on sequences, materials and methods, and additional experiments. (PDF)

\section{AUTHOR INFORMATION}

\section{Corresponding Authors}

*E-mail: efranco@engr.ucr.edu. Phone: +1-(951)-827-2442.

*E-mail: francesco.ricci@uniroma2.it. Phone: +39-0672594422.

\section{ORCID $\odot$}

Francesco Ricci: 0000-0003-4941-8646

Elisa Franco: 0000-0003-1103-2668

\section{Author Contributions}

"L.G. and A.A. contributed equally.

\section{Notes}

The authors declare no competing financial interest.

\section{ACKNOWLEDGMENTS}

All experiments conducted at the University of Rome were supported by the Associazione Italiana per la Ricerca sul Cancro, AIRC (project no. 14420) (F.R.), by the European Research Council, ERC (project no. 336493) (F.R.), and by the Italian Ministry of Education, University and Research (PRIN) (F.R.). All experiments conducted at UC Riverside as well as the salary for L.N.G. and H.K.K.S. were supported by the U.S. Department of Energy, Office of Science, Office of Basic Energy Sciences, under award no. DESC0010595 (E.F.).

\section{REFERENCES}

(1) Mann, S. Angew. Chem., Int. Ed. 2008, 47, 5306-5320.

(2) Pinheiro, A. V.; Han, D.; Shih, W. M.; Yan, H. Nat. Nanotechnol. 2011, 6, 763-772.

(3) Blind, M.; Blank, M. Mol. Ther.Nucleic Acids 2015, 4, 223.

(4) Zhang, D. Y.; Seelig, G. Nat. Chem. 2011, 3, 103-113.

(5) Fu, T. J.; Seeman, N. C. Biochemistry 1993, 32, 3211-3220.

(6) Winfree, E.; Liu, F.; Wenzler, L. A.; Seeman, N. C. Nature 1998, 394, 539-544.

(7) Rothemund, P. W.; Ekani-Nkodo, A.; Papadakis, N.; Kumar, A.; Fygenson, D. K.; Winfree, E. J. Am. Chem. Soc. 2004, 126, 1634416352.

(8) Ekani-Nkodo, A.; Kumar, A.; Fygenson, D. K. Phys. Rev. Lett. 2004, 93, 268301.

(9) Mitchell, J. C.; Harris, J. R.; Malo, J.; Bath, J.; Turberfield, A. J. J. Am. Chem. Soc. 2004, 126, 16342-16343.

(10) Liu, Y.; Lin, C.; Li, H.; Yan, H. Angew. Chem. 2005, 117, 44074412

(11) Sharma, J.; Chhabra, R.; Cheng, A.; Brownell, J.; Liu, Y.; Yan, H. Science 2009, 323, 112-116.

(12) Hariadi, R. F.; Appukutty, A. J.; Sivaramakrishnan, S. ACS Nano 2016, 10, 8281-8288.

(13) Kuzyk, A.; Schreiber, R.; Fan, Z.; Pardatscher, G.; Roller, E. M.; Högele, A.; Liedl, T.; Simmel, F. C.; Govorov, A. O. Nature 2012, 483, 311-314.

(14) Lin, J. L.; Wheeldon, I. ACS Catal. 2013, 3, 560-564.

(15) Stewart, J. M.; Viard, M.; Subramanian, H. K.; Roark, B. K.; Afonin, K. A.; Franco, E. Nanoscale 2016, 8, 17542-17550.

(16) Yang, Y.; Endo, M.; Hidaka, K.; Sugiyama, H. J. Am. Chem. Soc. 2012, 134, 20645-20653.

(17) Kamiya, Y.; Asanuma, H. Acc. Chem. Res. 2014, 47, 1663-1672.

(18) Liu, X.; Lu, C. H.; Willner, I. Acc. Chem. Res. 2014, 47, 16731680.

(19) Wang, F.; Lu, C. H.; Willner, I. Chem. Rev. 2014, 114, 28812941.

(20) Casey, J. R.; Grinstein, S.; Orlowski, J. Nat. Rev. Mol. Cell Biol. 2010, 11, 50-61.
(21) Whitten, S. T.; Garcia-Moreno, E. B.; Hilser, V. J. Proc. Natl. Acad. Sci. U. S. A. 2005, 102, 4282-4287.

(22) Gao, W.; Chan, J.; Farokhzad, O. C. Mol. Pharmaceutics 2010, 7, $1913-1920$.

(23) Grossmann, T. N.; Röglin, L.; Seitz, O. Angew. Chem., Int. Ed. 2007, 46, 5223-5225.

(24) Zheng, J.; Li, J.; Jiang, Y.; Jin, J.; Wang, K.; Yang, R.; Tan, W. Anal. Chem. 2011, 83, 6586-6592.

(25) Idili, A.; Plaxco, K. W.; Vallée-Bélisle, A.; Ricci, F. ACS Nano 2013, 7, 10863-10869.

(26) Liao, W. C.; Riutin, M.; Parak, W. J.; Willner, I. ACS Nano 2016, $10,8683-8689$

(27) Kahn, J. S.; Freage, L.; Enkin, N.; Garcia, M. A. A.; Willner, I. Adv. Mater. 2017, 29, 1602782.

(28) Hu, Y.; Ren, J.; Lu, C. H.; Willner, I. Nano Lett. 2016, 16, 45904594.

(29) Porchetta, A.; Idili, A.; Vallée-Bélisle, A.; Ricci, F. Nano Lett. 2015, 15, 4467-4471.

(30) Hu, Y.; Lu, C. H.; Guo, W.; Aleman-Garcia, M. A.; Ren, J.; Willner, I. Adv. Funct. Mater. 2015, 25, 6867-6874.

(31) Han, M. S.; Lytton-Jean, A. K.; Mirkin, C. A. J. Am. Chem. Soc. 2006, 128, 4954-4955.

(32) Guerrini, L.; McKenzie, F.; Wark, A. W.; Faulds, K.; Graham, D. Chem. Sci. 2012, 3, 2262.

(33) Amodio, A.; Adedeji, A. F.; Castronovo, M.; Franco, E.; Ricci, F. J. Am. Chem. Soc. 2016, 138, 12735-12738.

(34) Zhang, D. Y.; Hariadi, R. F.; Choi, H. M.; Winfree, E. Nat. Commun. 2013, 4, 1965.

(35) Green, L. N.; Subramanian, H. K. K.; Mardanlou, V.; Kim, J.; Hariadi, R. F.; Franco, E. In preparation, 2017. Downloadable at: https://tinyurl.com/y82oug87.

(36) Yurke, B.; Mills, A. P., Jr. Genetic Programming and Evolvable Machines 2003, 4, 111-122.

(37) Mohammed, A. M.; Schulman, R. Nano Lett. 2013, 13, 40064013.

(38) O’Brien, M. N.; Jones, M. R.; Lee, B.; Mirkin, C. A. Nat. Mater. 2015, 14, 833-839.

(39) Hadorn, M.; Boenzli, E.; Sørensen, K. T.; Fellermann, H.; Hotz, P. E.; Hanczyc, M. M. Proc. Natl. Acad. Sci. U. S. A. 2012, 109, 2032020325. 